\title{
Calidad de la docencia en enfermería: un concepto desde la percepción de sus actores
}

\author{
Paz Moscoso
}

Introducción. Los procesos de acreditación, necesarios en el actual escenario de la educación, requieren una descripción de los conceptos de calidad como un parámetro básico desde donde se deriven dimensiones e indicadores evaluables. Tomando en cuenta la polisemia del concepto de calidad, parece arriesgado que las descripciones de dicho concepto se generen de forma arbitraria. Respondiendo a este riesgo y carencia de conceptos, el objetivo del presente estudio cualitativo es generar colectivamente una conceptualización de calidad de la docencia en enfermería.

Sujetos y métodos. El surgimiento de conceptos que implica este estudio se logra a partir de la utilización de la teoría fundamentada. Las percepciones se obtuvieron mediante entrevistas en profundidad y grupos focales a docentes, estudiantes y licenciadas de enfermería, más el análisis recursivo de los datos obtenidos.

Resultados. Los elementos constituyentes de la conceptualización de la calidad de la docencia en enfermería incluyen, entre otros: valores del docente, habilidades de comunicación interpersonal docente-estudiante, características particulares del modelamiento del quehacer enfermero y metodologías de enseñanza en enfermería.

Conclusiones. Al obtener la conceptualización de la calidad de la docencia en enfermería desde las percepciones de los propios actores, su utilización en procesos de acreditación y evaluación (y en los cambios consecuentes que se puedan generar a partir de ello) podría ser aceptada de mejor forma por los mismos actores. Esto ayudaría, a su vez, a fortalecer los fundamentos de la disciplina.

Palabras clave. Calidad de la atención de salud. Cuidados de enfermería. Educación. Enfermería.

\section{Quality of teaching in Nursing: a concept from the perception of the actors}

Introduction. Accreditation processes, necessary as they are for the actual stage of education, require a description of quality concept as the basic parameter from which dimension and valuable indicators are to be derived. Considering the polysemy of the concept of quality, it seems risky that descriptions of such a concept were generated arbitrarily. Responsive to this risk and conceptual deficiency, the purpose of this qualitative study is to collectively generate a quality conceptualization of nursing teaching.

Subjects and methods. The emergence of concepts that the study reveals is accomplished by the application of grounded theory method. The reported perceptions were obtained in substantive interviews and focus groups teachers, students and Nursing graduated students, and the recursive analysis of the data.

Results. The constitutive elements of Nursing teaching quality conceptualization includes, among others: values of Nursing teachers; abilities of interpersonal communication between teacher and student; particulars characteristics of modelling the Nursing work; and methodologies of teaching in Nursing.

Conclusions. As we obtain the quality conceptualization in Nursing teaching from the perspective of the actors involved, its application in accreditation and evaluation processes (and the consequent changes generated from these), could be accepted in a better way by the same actors. And this could reinforced the foundations of the discipline.

Key words. Education. Nursing. Nursing care. Quality of health care.
Escuela de Enfermería. Universidad de los Andes. Las Condes, Santiago, Chile.

Correspondencia: Carmen Paz Moscoso Pavez. Escuela de Enfermería. Universidad de los Andes. San Carlos de Apoquindo, 2200 Las Condes, Santiago, Chile.

E-mail:

pazmoscoso@uandes.cl

Investigación realizada en el marco de la obtención del Máster en Educación en Ciencias de la Salud de la Universidad de Chile.

Conflicto de intereses: No declarado.

Competing interests: None declared.

(c) 2013 FEM 


\section{Introducción}

El actual contexto de la globalización del conocimiento ha involucrado el surgimiento de nuevos paradigmas pedagógicos, una nueva visión de la educación y múltiples modos de acceso al conocimiento. Frente a esto, y con el fin de garantizar calidad en la educación para la sociedad, surge la necesidad de regulación. Y con ello, los procesos de acreditación.

Dichos procesos establecen mediciones constantes de calidad en educación, incluyendo en ellas las evaluaciones de la calidad en la docencia. Cuando se realizan mediciones de calidad, y con el fin de tener un parámetro de referencias, es necesario definir dicho concepto y conocer sus componentes. Ahora bien, si consideramos que la conceptualización de la calidad de la docencia no es una abstracción, sino más bien un referente social e institucional [1], parece necesaria la búsqueda de una conceptualización que represente a ese grupo social o institucional.

En Chile, la enfermería está en una situación particular, pues ya desde 2003 se han iniciado las acreditaciones de las escuelas de enfermería, aun existiendo una carencia de la conceptualización de la calidad de la docencia en dicha disciplina [2].

La presente investigación utiliza como medio la teoría fundamentada, 'método cualitativo que mediante la recogida y análisis sistemático de datos, que surgen desde un colectivo particular, persigue generar nuevos conceptos' [3]. Con ella se intenta originar una conceptualización de calidad de la docencia en enfermería desde las percepciones de los actores involucrados, estudiantes, docentes y egresadas de la escuela de enfermería de la Universidad de los Andes.

Basándose en sus 13 años de experiencia en docencia en enfermería, la investigadora estima que si se estudian las percepciones y nociones de calidad de la docencia en enfermería en las estudiantes, docentes y licenciadas, es posible obtener una conceptualización en torno a dicha noción y extraer de los resultados los datos para el perfeccionamiento continuo en la labor.

Ahora bien, existe una conceptualización de la calidad que interpreta dicha noción como un elemento de rendimiento de mercado, y no en su dimensión humana. La docencia está en el centro del desarrollo humano, por lo que parece primordial que esa conceptualización se desvele desde las percepciones de las personas. Una noción que, a fin de cuentas, pueda acercarse a la noción de humana calidad.

Esta búsqueda se apoya en la idea de que el concepto de calidad utilizado en las instituciones debe corresponder a las necesidades particulares, como lo es la naturaleza de la disciplina (en este caso una disciplina que se ocupa del cuidado de la salud de las personas), y que también debe responder a las expectativas de docentes y estudiantes, que en este caso corresponde al colectivo del estudio [4].

Para mejorar la docencia, y en particular la docencia en enfermería, es necesario tener claro cuáles son los elementos que se valoran en el proceso para luego hacer énfasis en ellos. Creemos que dichos elementos deben surgir de las personas que desarrollan la actividad, pues no parece adecuado que se generen estándares desde expertos externos. Si surgen desde las personas podrían ser más valorados y, en el momento de necesitar implementar instancias para mejorar la calidad, la aceptación y la participación podrían ser mayores y con mejores efectos para la profesión y para la comunidad.

El objetivo general de la presente investigación es, por consiguiente, generar colectivamente una conceptualización de calidad de la docencia en enfermería desde las percepciones de estudiantes, docentes y licenciadas de enfermería de la Universidad de los Andes. Para el cumplimiento de dicho objetivo general, los objetivos específicos son:

- Dar significación al contenido de las percepciones de las estudiantes y licenciadas en relación con el concepto de calidad en la docencia, a partir de la interpretación de las docentes.

- Dar significación al contenido de las percepciones de las docentes en relación al concepto de calidad en la docencia, a partir de la interpretación de las estudiantes.

- Desvelar los elementos que forman parte del significado de la calidad de la docencia en enfermería a partir de las percepciones de las estudiantes y docentes.

- Reconstruir los significados de calidad de la docencia en enfermería, a partir de las percepciones de las estudiantes, licenciadas y docentes de enfermería de la Universidad de los Andes.

\section{Sujetos y métodos}

\section{Posicionamiento paradigmático}

Es importante recalcar que esta investigación valora la subjetividad como base en la resolución del problema. Este estudio se enmarca dentro del paradigma hermenéutico, el cual 'se ocupa de la comprensión del significado, ya que entiende que el conocimiento de las ciencias sociales no se puede desvincular de las estructuras de significación' [5]. 
El estudio valora cómo las personas -estudiantes, licenciadas y docentes- demuestran y dan significación a circunstancias que las rodean en la actividad diaria, ofreciendo una pauta o línea de reflexión tanto al docente sobre su actividad como al estudiante sobre su vinculación con el aprendizaje e inserción en la docencia. Esto supone, además, que no hay verdad instalada sobre el problema en estudio: el sujeto se apropia mediante su opinión de los conceptos, los resignifica a la luz de su propia experiencia, interpretando su propia realidad en vinculación con otras personas en un medio particular.

\section{Metodología}

La necesidad de generar una conceptualización de la calidad de la docencia en enfermería desde los propios actores requiere un método de investigación cualitativa que evidencie la teoría y los conceptos existentes sobre el particular y en un contexto social y cultural determinado.

Por este motivo, la investigación utiliza un método que recoge las percepciones y genera teoría desde dichas percepciones, develando así nuevos conceptos. El sustento metodológico de este trabajo lo constituye la teoría fundamentada, 'la cual orienta a descubrir teorías, conceptos, hipótesis y proposiciones partiendo directamente de los datos, no de los supuestos a priori, que surgen de otras investigaciones o de marcos teóricos o imposiciones convencionales' [6].

Se intenta llegar al concepto de la calidad de la docencia en enfermería como teoría derivada de los datos recopilados de forma sistemática. Como lo plantea la teoría fundamentada, en este caso no se parte de una preconcepción teórica, sino que se inicia con una área de estudio: la docencia en enfermería. De esta área interesa ver el concepto de calidad existente en esta disciplina en un colectivo específico de personas.

Con este método se persigue que el concepto obtenido desde el colectivo de personas genere conocimientos, aumente la comprensión y proporcione una guía validada para la acción en cuanto a la docencia en enfermería [7].

\section{Personas involucradas en la investigación}

Se intenta representar con la máxima fidelidad a todos los actores involucrados, por lo cual, en este caso, las personas representantes del colectivo son: docentes de profesión enfermera con más de cinco años de experiencia docente, estudiantes de los tres años finales de la carrera y licenciadas de enferme-
Tabla I. Dimensiones y categorías para codificación y análisis de datos. Matriz cualitativa 1: dimensiones y categorías para codificación y análisis de datos.

\begin{tabular}{|c|c|}
\hline Dimensiones & Categorías \\
\hline \multirow{3}{*}{$\begin{array}{l}\text { Características } \\
\text { del docente }\end{array}$} & Valores del docente \\
\hline & Habilidades en la relación profesor-estudiante \\
\hline & Modelamiento del quehacer enfermero \\
\hline \multirow{5}{*}{$\begin{array}{l}\text { Rasgos distintivos } \\
\text { de la docencia } \\
\text { en enfermería } \\
\text { en la institución }\end{array}$} & Valores \\
\hline & $\begin{array}{l}\text { Metodologías utilizadas en el } \\
\text { proceso de enseñanza-aprendizaje }\end{array}$ \\
\hline & Infraestructura \\
\hline & $\begin{array}{l}\text { Formación con principios católicos } \\
\text { (categoría emergente) }\end{array}$ \\
\hline & $\begin{array}{l}\text { Formación de la actitud profesional como parte } \\
\text { del profesionalismo (categoría emergente) }\end{array}$ \\
\hline \multirow{3}{*}{$\begin{array}{l}\text { Problemas } \\
\text { percibidos }\end{array}$} & Infraestructura \\
\hline & Gestión para la docencia \\
\hline & Proceso enseñanza-aprendizaje en enfermería \\
\hline \multirow{2}{*}{$\begin{array}{l}\text { Aseguramiento } \\
\text { de la calidad } \\
\text { de la docencia }\end{array}$} & Mantenimiento de rasgos distintivos de la escuela \\
\hline & $\begin{array}{l}\text { Estrategias de mejora de la } \\
\text { didáctica general y específica }\end{array}$ \\
\hline
\end{tabular}

ría de la Universidad de los Andes. A dichos actores se les incluirá en entrevistas y grupos focales.

Considérese correcta la expresión 'licenciadas, puesto que a la fecha del término de la investigación sólo existían personas de sexo femenino que habían terminado la carrera. Como criterio de inclusión común tenemos la voluntariedad con consentimiento informado.

\section{Recogida de información y análisis de los datos}

En una primera fase se realizaron entrevistas en profundidad para conocer las percepciones en relación al concepto de calidad de la docencia en enfermería a docentes informantes claves (seis entrevistadas), estudiantes informantes claves (cuatro entrevistadas) y licenciadas informantes claves (cinco entrevistadas). El número de entrevistadas estuvo sujeto a la saturación de la información obtenida.

Tras la transcripción total de estas entrevistas se envió la transcripción a cada una de las entrevista- 
Tabla II. Análisis de las interpretaciones de las estudiantes acerca de los relatos de las docentes (sólo incluye página 1 de matriz para ejemplificar). Matriz cualitativa 3: análisis de interpretaciones de las estudiantes acerca de los relatos de las docentes.

\begin{tabular}{|c|c|c|}
\hline Dimensiones & Categorías & Interpretación de las estudiantes (grupo focal) \\
\hline \multirow{5}{*}{$\begin{array}{l}\text { Características } \\
\text { del docente }\end{array}$} & \multirow{2}{*}{ Valores del docente } & $\begin{array}{l}\text { Las estudiantes consideran valiosos los elementos destacados por las docentes, como el valor } \\
\text { del ser humano, el compromiso: 'Uno puede mandar un e-mail a las } 19 \mathrm{~h} \text { y a las } 20 \text { h está } \\
\text { respondido [...] uno sabe que es fuera del horario de trabajo' (ETF 1) }\end{array}$ \\
\hline & & $\begin{array}{l}\text { También reconocen el respeto y la colaboración entre los docentes, aunque reconocen que no } \\
\text { se da en todas las docentes: 'Como perfil de docentes lo tienen, pero en ciertas circunstancias } \\
\text { no se ha cumplido' (ETF 3) }\end{array}$ \\
\hline & \multirow{2}{*}{$\begin{array}{l}\text { Habilidades en la relación } \\
\text { profesor-estudiante }\end{array}$} & $\begin{array}{l}\text { Las estudiantes tienen plena coincidencia con los relatos sobre la relación profesor-estudiante; } \\
\text { destaca en ello la cercanía percibida y lo contrastan con experiencias vividas en los campos } \\
\text { clínicos: 'En clínica me he dado cuenta de que la relación en general es muy distinta de la } \\
\text { profesora de los Andes con sus alumnas, que las de la Universidad X con sus alumnas' (ETF 3) }\end{array}$ \\
\hline & & $\begin{array}{l}\text { Además, reconocen la relación docente-estudiante que se da en el lugar como un elemento } \\
\text { favorecedor del aprendizaje: 'la mezcla de exigencia y de cariño te dan ganas de aprender, } \\
\text { se nota y se agradece' (ECF 2) }\end{array}$ \\
\hline & $\begin{array}{l}\text { Modelamiento del } \\
\text { quehacer enfermero }\end{array}$ & $\begin{array}{l}\text { En el grupo hay consenso sobre los relatos de las docentes en relación al modelamiento del } \\
\text { quehacer enfermero, en los cuales, según las docentes, destacan la preocupación por los detalles, } \\
\text { el modelo de atención en el campo clínico: 'uno anda mirando los detalles' (ECF 3), 'tenemos } \\
\text { superbuenos modelos, además uno va al campo clínico de inmediato' (EQF 2) }\end{array}$ \\
\hline
\end{tabular}

ETF: estudiante de tercer año en grupo focal; ECF: estudiante de cuarto año en grupo focal; EQF: estudiante de quinto año en grupo focal; DGF: docente en grupo focal; es: estudiantes (en entrevistas en profundidad); eg: licenciadas (en entrevistas en profundidad); doc: docentes (en entrevistas en profundidad).

das para validar el escrito y ofrecer la oportunidad de dar nuevos aportes o nuevas reflexiones (comparación constante con informantes). Luego, 'se iniciará el 'microanálisis' [para] examinar e interpretar los datos de manera minuciosa' [7]. Con este análisis se llevó a cabo la primera generación de dimensiones y categorías (Tabla I). No se realizó una descripción categorial previa a las entrevistas, pues se espera que surjan desde el colectivo estudiado. Recién con las primeras entrevistas codificadas se inició el proceso de determinación categorial inicial, que se realizó mediante análisis estructural de las entrevistas. Se sintetizaron estos datos y se obtuvo una segunda matriz cualitativa (Tabla II).

Tras la generación de las dimensiones y las categorías iniciales originadas del análisis estructural de entrevistas en profundidad, se realizaron dos grupos focales:

- En el grupo focal con los estudiantes (diez participantes) se expuso el análisis de las entrevistas en profundidad a docentes. Se recogió entonces la interpretación que ellos lograron respecto al contenido de las percepciones de las docentes. Con esto, la investigadora analizó nuevamente el texto mediante la transcripción y el análisis estructural del desarrollo del grupo focal, para dar así una significación a este contenido en virtud del objetivo final de la investigación. Se sintetizaron estos datos y se obtuvo una tercera matriz cualitativa (Tabla II).

- En el grupo focal con las docentes (seis participantes) se expuso el análisis inicial de las entrevistas en profundidad a estudiantes y licenciadas. Se recogió entonces la interpretación que lograron respecto al contenido de las percepciones de los estudiantes y licenciadas. Con ello, la investigadora analizó nuevamente el texto, realizando comparación (constante) entre el contenido de las entrevistas, ya codificadas, y el contenido de los relatos obtenidos en el grupo focal, con el fin de delinear los contenidos en virtud del objetivo final de la investigación. Se sintetizaron estos datos y se obtuvo una cuarta matriz cualitativa.

Tras ambos grupos focales se continuó enriqueciendo el sistema categorial mediante la comparación constante de los textos obtenidos luego de las transcripciones.

Se realizó entonces un nuevo análisis de los datos ya reducidos, haciendo comparación de la información obtenida desde las entrevistas y desde 
los grupos focales. Se sintetizaron estos datos y se realizó una matriz cualitativa integradora, la cual considera los textos de todas las matrices anteriores. De este análisis no surgió la necesidad de reunirse nuevamente con los actores involucrados.

Con la información obtenida se llevó a cabo entonces la fase de interpretación de los datos por parte de la investigadora, mediante lo cual se llegó a la generación de una conceptualización del concepto de la calidad de la docencia en enfermería que incluye una descripción de ella y una enumeración de los elementos constitutivos de la docencia en enfermería en la escuela de enfermería de la Universidad de los Andes.

Esta conceptualización de la calidad de la docencia en enfermería fue reconstruida en base a los discursos de todos los actores, tanto en entrevistas como en grupos focales, incluyendo, durante todo el tiempo que duró la investigación, las percepciones de la investigadora respecto al concepto de calidad vivenciado en los años de docencia en la institución donde se desarrolla el estudio.

Luego se procedió a la comunicación de los resultados a actores involucrados seleccionados al azar (dos estudiantes, dos docentes, dos licenciadas), a quienes se les pidió opinión respecto a los hallazgos para nuevas aportaciones y validación de lo obtenido. No hubo nuevas aportaciones.

Las fases seguidas y descritas anteriormente se resumen en la figura 1.

\section{Resultados}

\section{Matrices cualitativas}

Del proceso de recogida y análisis recursivo de los datos se obtienen las matrices cualitativas que expresan los resultados obtenidos (ej., Tabla II).

\section{Componentes de la conceptualización de la calidad de la docencia en enfermería del colectivo estudiado}

Los elementos constitutivos de la calidad de la docencia en enfermería (Fig. 2) surgen del análisis de las matrices cualitativas por parte de la investigadora y no corresponden directamente al sistema categorial de análisis de datos, sino más bien a una reorganización de las dimensiones y categorías expuestas en las matrices, derivada del análisis y comparación con el marco teórico de la investigación.

A modo de ejemplo señalamos a continuación segmentos de textos obtenidos en entrevistas o grupos focales que son representativos de algunos de
Figura 1. Secuencia metodológica de recogida y análisis recursivo de los datos.

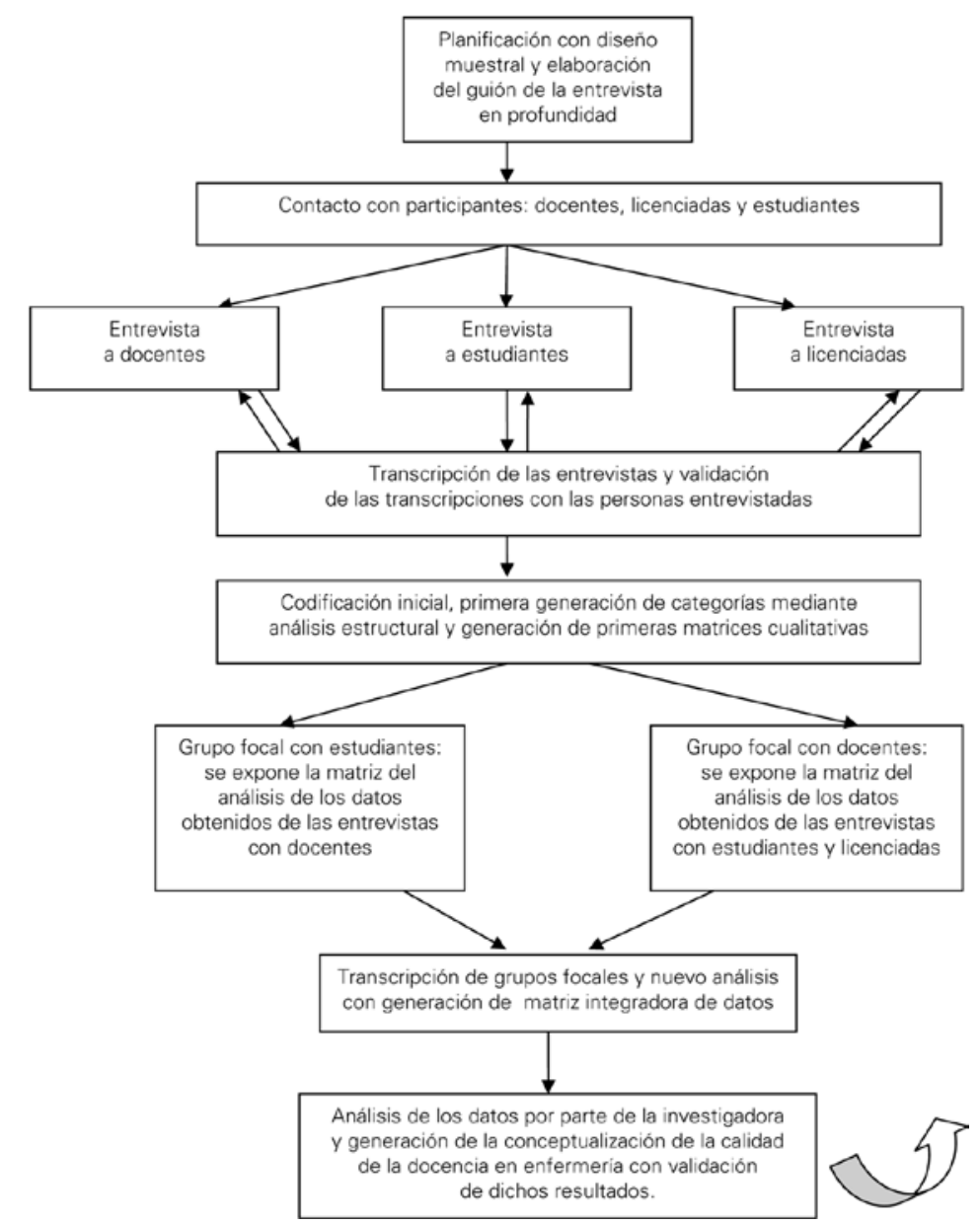

los elementos constitutivos del concepto de calidad de la docencia en enfermería.

\section{Respeto por el ser humano}

- 'De parte de las docentes me llevaré que las docentes son muy humanas, se distinguen en el modo como nos tratan y como tratan a los pacientes' (es 3).

- 'Yo creo que uno tiene internalizado el valor que tiene el ser humano' (doc 1).

\section{Confianza y acompañamiento}

- '[...] varias docentes tienen un rol muy maternal con uno; si uno se equivoca, en lugar de reprimir, te hacen una acogida' (eg 2).

- '[...] hay un rol más acogedor con el error' (eg 2).

- 'Tienen la cualidad de estar contigo, al lado' (es 1). 
Figura 2. Elementos constitutivos de la calidad de la docencia en enfermería.

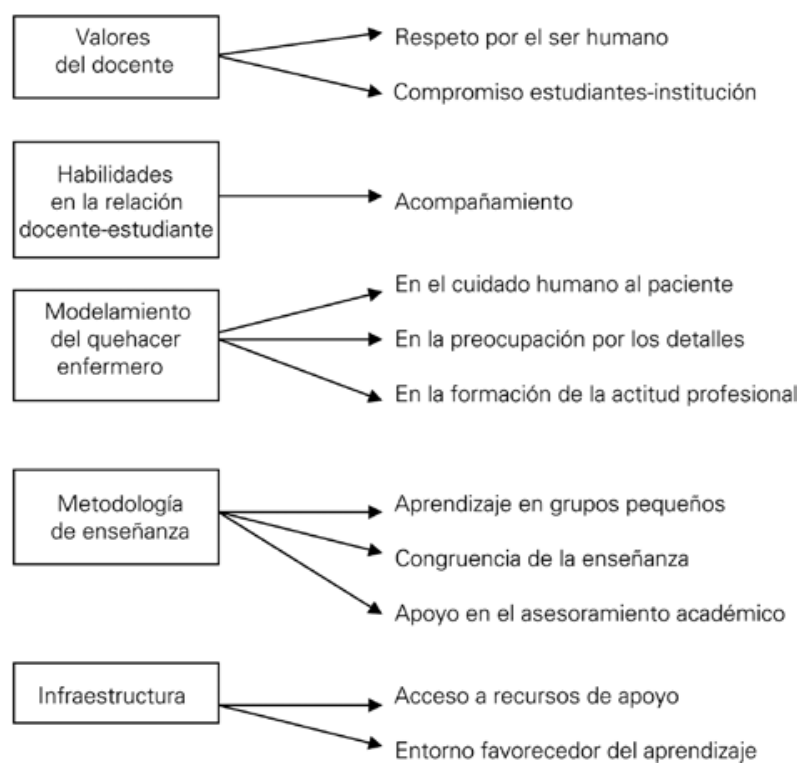

- '[...] la mezcla de exigencia y de cariño te da ganas de aprender, se nota y se agradece' (ECF 2).

\section{Modelamiento del quehacer enfermero en el cuidado humano al paciente}

'[...] compartimos que lo primero es el paciente y la familia, y lo compartimos como un valor, no como un discurso bonito [...]. Cuando vas a clínica, las alumnas lo ven, es un modelo que a las alumnas se les impregna' (doc 4).

\section{Congruencia de la enseñanza del aula al campo clínico}

- 'Hacen de profesoras aquí [universidad] y luego en la clínica eran las mismas, eso es bueno' (eg 1).

- 'Todas [las docentes] van al campo clínico y es importante porque los conocimientos son coherentes. No se contradicen, y eso es un beneficio. Sería muy raro que no fueran' (es 4).

\section{Reconstrucción de la conceptualización de calidad de la docencia en enfermería}

A continuación se reconstruye el concepto de calidad de la docencia en enfermería en base a la lectura cuidadosa y el análisis de los discursos generados en entrevistas, grupos focales (entre paréntesis se cita a todos los involucrados en el estudio): 'Activi- dad docente centrada en el valor y la dignidad del ser humano. Esta actividad destaca por el respeto y el valor dado al estudiante, mediante un trabajo cercano y personalizado que permite a éste reconocer su propio valor como persona $y$, consecuentemente, proyectarlo al cuidado efectivo e integral de la salud de las personas' (Inf.: AP, AM, RV, NH, PB, PV, BH, MA, DI, PV, IN, PJ, XV, AG, GE, CG, TA, CO, VN, PI, AM, PR, CJ, MU, DD, FA, AE, LS, XL, BT, GE. 2009-2010).

\section{Discusión}

\section{Significado y aplicación práctica de los resultados}

Este estudio refleja una autoevaluación inicial del quehacer en la escuela de enfermería de la Universidad de los Andes, que es una primera y valiosa instancia para mejorar algunas áreas que presentan carencias y reforzar los rasgos que hoy son valorados como distintivos.

Los resultados a los cuales ha llegado este estudio pueden ofrecer sustento para la generación de estándares de calidad de la docencia en enfermería en la Escuela de Enfermería de la Universidad de los Andes, demostrando una identidad particular en la enseñanza de la enfermería.

Los elementos constituyentes de la conceptualización de la calidad de la docencia en enfermería podrían ser de utilidad a otras escuelas de enfermería, pues reflejan el eje de la actividad de la docencia en esta área, que es el cuidado de la persona.

Considerando los antecedentes logrados y la consecuente conceptualización generada, esbozamos a continuación una primera enumeración de dimensiones para la evaluación de la calidad de la docencia en enfermería:

- Dimensión 1: comunicación e interacción docente-estudiante.

- Dimensión 2: metodología de enseñanza-aprendizaje.

- Dimensión 3: contenidos del aprendizaje en enfermería.

- Dimensión 4: recursos necesarios para el aprendizaje (la descripción de las dimensiones puede solicitarse a la autora).

\section{Relación con publicaciones similares y contraste con la teoría}

Como se ha mencionado en la introducción, en la teoría hay carencia de determinación del concepto de calidad de la docencia y de los componentes de 
ella desde los propios actores. Aun así, existen elementos teóricos descritos por expertos en educación que, a continuación, se vinculan a modo de discusión con la investigación presentada.

\section{Concepción y enfoque del aprendizaje}

Teniendo en cuenta el papel del docente y la orientación de la educación, que forma parte de la conceptualización de la calidad de la docencia según las percepciones de docentes, licenciadas y estudiantes de la Escuela de Enfermería de la Universidad de los Andes, podemos afirmar que en ellos se trasuntan lineamientos de teorías del aprendizaje; aunque está claro que las teorías del aprendizaje son aproximaciones parciales y restringidas a áreas concretas del aprendizaje [8], podemos vincular los resultados obtenidos con la teoría constructivista del aprendizaje, pues en el constructivismo el estudiante construye el conocimiento de manera personal mediada por la intervención del profesor, en un momento y contexto cultural particulares, como lo es en el caso del colectivo estudiado, inserto en un contexto particular. Además, los resultados son coherentes con los fines definidos en la misión de la institución y el perfil docente descrito, y la construcción del conocimiento tiene una orientación concreta hacia metas definidas [9]. El estudiante es responsable último de su proceso de aprendizaje [10], tal como se reconoce en los relatos de los docentes.

El constructivismo además amerita que los docentes valoren y promuevan la actuación autónoma del estudiante, generando prácticas educativas que potencien dicha construcción [11]. Como vemos en este estudio, la docencia ejercida en la escuela de enfermería estudiada promueve la iniciación de actividades en campos clínicos en el primer año de la carrera, lo que favorece la inserción precoz en el lugar donde se desarrolla la profesión. De esta forma, el estudiante logra dar sentido a sus estudios, orientando entonces un aprendizaje más significativo.

Considerando que tanto las declaraciones de Flórez [12] como los fines de la educación descritos por Delors [13] coinciden en que el verdadero aprendizaje es aquel que contribuye al desarrollo de la persona, hemos visto en los resultados que el docente de enfermería del estudio orienta el aprendizaje al cuidado del ser humano, centrándose previamente en el valor del estudiante como persona; apoya de forma cercana al estudiante, favoreciendo su propio desarrollo, y luego lo valora como persona que puede construir conocimientos sólidos en pos del cuidado del ser humano.
Tabla III. Paralelismos entre los hallazgos del estudio y elementos que se vinculan con la calidad de la docencia desarrollados por expertos.

\begin{tabular}{|c|c|}
\hline $\begin{array}{l}\text { Rasgos y elementos de una docencia de } \\
\text { calidad desarrollados por autores expertos }\end{array}$ & Hallazgos del estudio \\
\hline Entusiasmo, incluyendo fuerza y estilo [14] & $\begin{array}{l}\text { 'Tiene [...] ganas de explicártelas } \\
\text { y además lo explican bien' (es 1) }\end{array}$ \\
\hline Empatía, mostrando interés por los estudiantes [14] & 'Hay un papel más acogedor con el error' (eg 2) \\
\hline \multirow[t]{2}{*}{ Atención personal a los estudiantes [15] } & $\begin{array}{l}\text { 'El asesoramiento académico [...] apunta a ayudar a } \\
\text { la alumna a desarrollar mejor sus potencialidades } \\
\text { [...]; uno los guía, que se sienta respaldado' (doc 1) }\end{array}$ \\
\hline & 'Uno es más que un número, es una persona' (ETF 2) \\
\hline Los profesores excepcionales esperan 'más' [17] & $\begin{array}{l}\text { 'Nosotros somos unos meros orientadores, } \\
\text { pero el trabajo es de los alumnos' (doc 5) }\end{array}$ \\
\hline $\begin{array}{l}\text { Los profesores muy efectivos tienden a mostrar } \\
\text { una gran confianza en los estudiantes [17] }\end{array}$ & $\begin{array}{l}\text { '[...] tratando de ver los aspectos positivos, } \\
\text { las cualidades de cada uno' (doc } 5 \text { ) }\end{array}$ \\
\hline $\begin{array}{l}\text { Una conducta docente interactiva que } \\
\text { modela habilidades interpersonales [18] }\end{array}$ & $\begin{array}{l}\text { 'El ejemplo, cómo te tratan como persona y cómo } \\
\text { tratan las profesoras a los pacientes' (es } 2 \text { ) }\end{array}$ \\
\hline Facilitar el aprendizaje [16] & $\begin{array}{l}\text { 'La mezcla de exigencia y de cariño te da ganas } \\
\text { de aprender, se nota y se agradece' (ECF 2) }\end{array}$ \\
\hline $\begin{array}{l}\text { Facilitar el desarrollo y la socialización } \\
\text { de los estudiantes [16] }\end{array}$ & $\begin{array}{l}\text { ‘Uno se encuentra con pacientes con distintas } \\
\text { «formas» espirituales, pero uno acepta cualquier } \\
\text { tipo de religión [...]; uno lleva una vida espiritual } \\
\text { que al final al paciente le sirve de mucho' (eg 2) }\end{array}$ \\
\hline
\end{tabular}

\section{Debilidades de los hallazgos}

Si bien en los hallazgos del estudio nos encontramos, sobre todo, con grandes fortalezas (contenidas principalmente en la transmisión de valores, que trasuntan todas las actividades vinculadas con la docencia), es posible reconocer una falta de evidencia en los relatos de estudiantes, e incluso de docentes, acerca de elementos vinculados con la evaluación del proceso de enseñanza-aprendizaje, necesarios para el logro de la calidad en la docencia. Algunas de estas debilidades son: procedimientos de evaluación incluyendo la calidad del feedback [14], mecanismos de revisión del proceso [15] y utilización y evaluación de las estrategias de evaluación [16]

A modo de resumen de la discusión de los resultados presentamos un esquema con paralelismos entre los hallazgos del estudio y elementos que se vinculan con la calidad de la docencia desarrollados por expertos (Tabla III).

\section{Bibliografía}

1. Salas-Perea R. La calidad en el desarrollo profesional: avances y desafíos. Rev Cubana Educ Med Super 2000; 14: 136-47. 
2. Caballero E. Estudio de fortalezas y debilidades de las escuelas de enfermería acreditadas por la CNAP. URL: http://www. achieen.cl. [02.05.2009].

3. Gerrish K. Investigación en enfermería. 5 ed. Madrid: McGraw-Hill; 2008

4. González L. Calidad de la educación superior: conceptos y modelos. Revista Calidad en la Educación 2008; 28: 248-76.

5. Ruiz de Gauna P. Documentos referenciados. Módulo seminario de tesis. Programa de Magíster en Educación en Ciencias de la Salud. DECSA. Santiago: Universidad de Chile; 2008.

6. Rodríguez G, Gil J, García E. Metodología de la investigación cualitativa. Málaga: Aljibe; 1996.

7. Corbin J, Strauss A. Bases de la investigación cualitativa. Técnicas y procedimientos para desarrollar la teoría fundamentada. Medellín: Universidad de Antioquia; 2002.

8. DECSA. Aprendizaje. Cómo hacer más efectivos y eficientes nuestros aprendizajes. Documentos referenciados. Módulo de aprendizaje. Programa de Magíster en Educación en Ciencias de la Salud. Santiago: Universidad de Chile; 2008.

9. Mauri T, Coll C, Onrubia J. La evaluación de la calidad de los procesos de innovación de la calidad de los procesos de innovación universitaria. Una perspectiva constructivista. Red U. Revista de Docencia Universitaria. URL: http://www. redu.um.es. [31.03.2010].
10. Coll S. Aprendizaje y construcción. 1 ed. Barcelona: Paidós; 1990.

11. Reyes W, González R. Aproximación de la fundamentación teórica de un modelo de formación docente constructivista. Omnia 2007; 13: 131-54.

12. Flórez R. Hacia una pedagogía del conocimiento. Santa Fé de Bogotá: McGraw-Hill; 1996.

13. Delors J. Informe de la UNESCO de la Comisión Internacional sobre la educación para el siglo XXI. La educación encierra un tesoro. Madrid: Santillana; 1996.

14. Marsh H. Students evaluations of university teaching research methods, methodological issues, and directions for future research. Int J Educ Res 1987; 11: 253-388.

15. Zabalza M. Competencias docentes del profesorado universitario: calidad y desarrollo profesional. Madrid: Narcea; 2003.

16. National League for Nursing. Core competences of nurse educators 2005. URL: http://www.nln.org. [24.03.2009].

17. Bain K. Lo que hacen los mejores profesores universitarios. 2 ed. Valencia: Universidad de Valencia; 2007.

18. Hillier Y, Vielba C. Perceptions of excellence: personal constructs of excellence in teaching and learning. Institute of Learning and Teaching Annual Conference. University of York 2001. URL: http://www.heacademy.ac.uk. [09.04.2010] 